\title{
Signalling pathway of isophorone diisocyanate-responsive interleukin-8 in airway smooth muscle cells
}

\author{
P-L. Kuo*,\#, M-S. Huang, , , S-K. Huang ${ }^{\#,+, \$, ~ W-C . ~ N i ~}{ }^{+}$, J-Y. Hung ${ }^{\#,{ }^{+},+}$, Y-C. Ko*,\#, \\ C-H. Hung ${ }^{\#, f}$, Y-M. Tsai ${ }^{\#, \oplus}$, T-H. Duh ${ }^{\#, * *}$ and Y-L. Hsu ${ }^{+}$
}

ABSTRACT: This study is the first to analyse the soluble factors secreted by the bronchial epithelium after exposure to isophorone diisocyanate (IPDI) that are responsible for increasing migration and proliferation of primary normal human bronchial smooth muscle cells (BSMCs).

We treated immortalised, nontumorigenic human bronchial epithelial cells (cell line BEAS-2B) and primary normal human bronchial epithelial cells (HBEC) with IPDI, and then collected the conditioned culture media (IPDI-BEAS-2B-CM and IPDI-HBEC-CM, respectively), which was added to BSMCs.

Exposure of BEAS-2B cells and HBECs to IPDI increased interleukin (IL)-8 production. Culture of BSMCs with IPDI-BEAS-2B-CM and IPDI-HBEC-CM increased BSMC proliferation and migration, which are major features in asthma-related airway remodelling. Induction of BSMC proliferation and migration by IPDI-BEAS-2B-CM and IPDI-HBEC-CM was associated with increased focal adhesion kinase (FAK), Src, extracellular signal-regulated kinase (ERK) $1 / 2$ and AKT activation. Blocking FAK with a specific inhibitor significantly decreased BSMC migration and proliferation by inhibiting ERK1/2 activation. FAK and ERK1/2 inhibitor also decreased IPDIBEAS-2B-CM-, IPDI-HBEC-CM- and recombinant human IL-8-mediated BSMC proliferation and migration, whereas blocking Rnd3 using small interfering RNA failed to affect BSMC proliferation, suggesting that Rnd3 was only involved in the regulation of BSMC migration.

Our study suggests that inhibition of IL-8 or IL-8-mediated FAK/ERK/Rnd3 signalling is an attractive therapeutic target for IPDI-mediated asthma.

KEYWORDS: Interleukin-8, isophorone diisocyanate, migration, occupational asthma, proliferation, Rnd3

xposure to isocyanates and other low molecular weight chemicals causes 5$15 \%$ of all occupational asthma, which is a major health problem [1]. Isophorone diisocyanate (IPDI), an aliphatic diisocyanate used to manufacture polyurethane plastics, has been reported to cause occupational adult asthma $[2,3]$. Characteristics of bronchial and lymph node biopsies from diisocyanate asthma patients and laboratory animals show pathological features similar to those seen in atopic asthma, including increased inflammatory and T-helper cell type 2 responses, and enhancement of airway remodelling $[1,4]$. The major features of airway remodelling include: loss of epithelial integrity, subepithelial fibrosis, goblet cell and submucosal gland enlargement, increased bronchial smooth muscle mass, and increased angiogenesis [5]. Human bronchial smooth muscle cells (BSMCs) play a key role in the modulation of airway tone. In an asthmatic airway, BSMCs not only increase secretory and proliferative ability, but also migrate to the subepithelial area $[5,6]$. In addition, BSMCs also release proinflammatory cytokines, which are responsible for the progression of asthma pathogenesis. The degree of change in bronchial smooth muscle mass has been correlated to asthma severity [6].

Focal adhesion kinase (FAK), a nonreceptor protein tyrosine kinase, is involved in mediation of signalling cascade and plays an important role in cell proliferation, survival, adhesion and movement [7, 8]. The activation of FAK is regulated by phosphorylation at the sixth tyrosine residue. Autophosphorylation of FAK at tyrosine 397 forms a binding site for Src, which in turn phosphorylates FAK at Tyr 576/577, which is essential for optimum FAK kinase activity. The FAK-Src complex also phosphorylates FAK at
AFFILIATIONS

*Institute of Clinical Medicine,

${ }^{\#}$ Center of Excellence for Environmental Medicine,

${ }^{+}$Graduate Institute of Medicine,

**Faculty of Medicinal and Applied Chemistry, Kaohsiung Medical University,

"Division of Pulmonary and Critical Care Medicine, and

fDepartment of Paediatrics, Kaohsiung Medical University Hospital, Kaohsiung, Taiwan. §.Johns Hopkins Asthma and Allergy Center, School of Medicine, Johns Hopkins University, Baltimore, MD, USA.

CORRESPONDENCE

Y-L. Hsu

Graduate Institute of Medicine Kaohsiung Medical University 100 Shih-Chuan 1st Road Kaohsiung 807

Taiwan

E-mail: hsuyl326@gmail.com

Received:

Dec 042009

Accepted after revision:

Aug 162010

First published online:

Sept 032010

European Respiratory Journa

Print ISSN 0903-1936

Online ISSN 1399-3003 
Tyr 925, which forms a docking site for the adaptor growth factor receptor-bound protein-2 and activates the mitogenactivated protein kinase (MAPK) pathway [9]. Rnd3/RhoE, a Rho guanosine triphosphatase, is activated by Raf-MEK-ERK [10], and has been reported to be involved in many cellular functions, including cell migration, proliferation, cell cycle progression, apoptosis and differentiation [11-13]. Overexpression of Rnd3/RhoE disrupts actin cytoskeleton organisation and focal adhesions in fibroblasts and epithelial cells [14]. For example, high expression of Rnd3 has been found to increase the invasive ability of melanoma [11], but the function of Rnd3 has been less well studied in BSMCs.

We hypothesised that IPDI may cause epithelial cells to produce the soluble factor(s) that, in turn, increase proliferation and migration of BSMCs. Therefore, we treated BSMC with IPDI-treated bronchial epithelial cell-conditioned culture medium, and assessed the mechanisms of BSMC proliferation and migration. This model provided evidence of the interaction between bronchial epithelial cells and BSMC, and the mechanism of BSMC migration after IPDI exposure.

\section{METHODS}

\section{Cell culture and conditioned media}

Immortalised, nontumorigenic human bronchial epithelial cells (cell line BEAS-2B; American Type Culture Collection number CRL-9609) were cultured in Dulbecco's modified Eagle's medium/F-12 supplemented with $10 \%$ heat-inactivated fetal bovine serum, $2 \mathrm{mM}$ L-glutamine, $100 \mathrm{U} \cdot \mathrm{mL}^{-1}$ penicillin and $100 \mu \mathrm{g} \cdot \mathrm{mL}^{-1}$ streptomycin at $37^{\circ} \mathrm{C}$ in a humidified atmosphere containing $5 \% \mathrm{CO}_{2}$. Primary normal human BSMCs and primary normal human bronchial epithelial cells (HBECs) were obtained from Lonza (Walkersville, MD, USA). BSMCs were cultured in Smooth Muscle Growth Medium 2 (Lonza) and HBECs were cultured in Bronchial Epithelial Cell Growth Medium (Lonza).

To obtain IPDI-treated BEAS-2B- and HBEC-conditioned media (IPDI-BEAS-2B-CM and IPDI-HBEC-CM, respectively), BEAS-2B cells and HBECs $\left(2 \times 10^{6}\right.$ cells per $100-\mathrm{mm}$ dish $)$ were treated with vehicle control or various concentrations of IPID for $6 \mathrm{~h}$. After treatment, the medium was replaced and the supernatants harvested after $24 \mathrm{~h}$ of incubation. Interleukin (IL)-8 depletion from IPDI-BEAS-2B-CM and IPDI-HBEC-CM was performed using anti-IL-8 antibodies $\left(2 \mu \mathrm{g} \cdot \mathrm{mL}^{-1}\right)$ and Sepharose A/G beads following standard immunoprecipitation protocols. Cytokine depletion was confirmed using an IL-8 ELISA assay kit (R\&D Systems Europe, Abingdon, UK).

\section{ELISA and cytokine arrays}

The levels of IL-8, CXC chemokine ligand (CXCL) 5 and IL-1 $\beta$ were determined using ELISA-based kits (R\&D Systems Europe). ELISAs were performed according to the manufacturer's instructions. The profile of cytokines expressed by IPDItreated BEAS-2B cells was also assessed by RayBio ${ }^{\circ}$ Human Inflammation Antibody Array (RayBiotech, Inc., Norcross, GA, USA) according to the manufacturer's instructions.

\section{Cell proliferation}

Cells $\left(4 \times 10^{3}\right.$ per well) were plated in 96-well culture plates. After $24 \mathrm{~h}$ incubation, the cells were treated with vehicle control conditioned medium, IPDI-BEAS-2B-CM or IPDI-HBEC-CM for
$72 \mathrm{~h}$. The proliferation of BSMCs was determined using Premixed WST-1 Cell Proliferation Reagent (Clontech Laboratories Inc., Mountain View, CA, USA) according to the manufacturer's instructions.

\section{Cell migration assay}

Cell migration was carried out using the QCM Chemotaxis 8- $\mu \mathrm{m}$ Cell Migration Assay System (Millipore Corp., Bedford, MA, USA) according to the manufacturer's instructions. Cells were seeded into the migration chamber, and IPDI-BEAS-2B-CM, IPDI-HBEC-CM, vehicle control conditioned medium, IL-8depleted IPDI-BEAS-2B-CM, IL-8-depleted IPDI-HBEC-CM or medium containing $20 \mathrm{ng} \cdot \mathrm{mL}^{-1}$ recombinant human (rh)IL-8 was placed in the lower chamber. After allowing cell migration for $24 \mathrm{~h}$, cells that had migrated through the membrane were stained, lysed and quantified on a microplate at $520 \mathrm{~nm}$.

\section{Immunoblotting and immunoprecipitation}

Cells were lysed on ice for 15 min usingy M-PER lysis reagent (Pierce, Rockford, IL, USA). Cell lysates were centrifuged at $14,000 \times g$ for $15 \mathrm{~min}$ and the supernatant fraction collected for immunoblotting. Equivalent amounts $\left(20 \mu \mathrm{g} \cdot \mathrm{mL}^{-1}\right)$ of protein were resolved by sodium dodecylsulfate-polyacrylamide gel electrophoresis $(8-12 \%)$ and transferred to polyvinylidene fluoride membranes. After blocking for $1 \mathrm{~h}$ in $5 \%$ nonfat dry milk in Tris-buffered saline, the membrane was incubated with primary antibody for 1-16 h. The membrane was then treated with the appropriate peroxidase-conjugated secondary antibody and the immunoreactive proteins detected using an enhanced chemiluminescence kit (Millipore) according to the manufacturer's instructions.

\section{Real-time RT-PCR and microarray}

RNA isolation was performed using the TRIzol reagent (Invitrogen, Carlsbad, CA, USA). cDNA was prepared using an oligodeoxythymidine primer and reverse transcriptase (Takara, Shiga, Japan) following standard protocols. Real-time PCR was performed by using SYBR Green on the ABI 7500 Real-Time PCR System (Applied Biosystems, Foster City, CA, USA). Each PCR reaction mixture contained $200 \mathrm{nM}$ of each primer, $10 \mu \mathrm{L}$ of $2 \times$ SYBR Green PCR Master Mix (Applied Biosystems), $5 \mu \mathrm{L}$ cDNA and ribonuclease-free water in a total volume of $20 \mu \mathrm{L}$. The PCR reaction was carried out with a denaturation step at $95^{\circ} \mathrm{C}$ for $10 \mathrm{~min}$, and then for 40 cycles at $95^{\circ} \mathrm{C}$ for $15 \mathrm{~s}$ and $60^{\circ} \mathrm{C}$ for $1 \mathrm{~min}$. All PCRs were performed in triplicate and normalised to internal control glyceraldehyde-3phosphate dehydrogenase (GAPDH) mRNA. Relative expression is presented as $2^{-\Delta \Delta C T}$, where $\Delta$ represents "change in" and CT represents concentration.

Microarray experiment procedures were carried out following the manufacturer's protocols. Total RNA $(1 \mu \mathrm{g})$ was amplified with an Agilent Quick Amp Labeling Kit (Agilent Technologies, Santa Clara, CA, USA). IPDI-BEAS-2B-CM- and IPDI-HBECCM-treated BSMC RNA was labelled with Cy5, and control conditioned medium-treated BSMC RNA was labelled with Cy3 in an in vitro transcription process. $0.825 \mu \mathrm{g}$ Cy-labelled cRNA was cleaved to an average size of about $50-100$ nucleotides by incubation with fragmentation buffer (Agilent Technologies) at $60^{\circ} \mathrm{C}$ for $30 \mathrm{~min}$. An equal amount of Cy-labelled cRNA was pooled and hybridised to an Agilent Whole Human Genome 

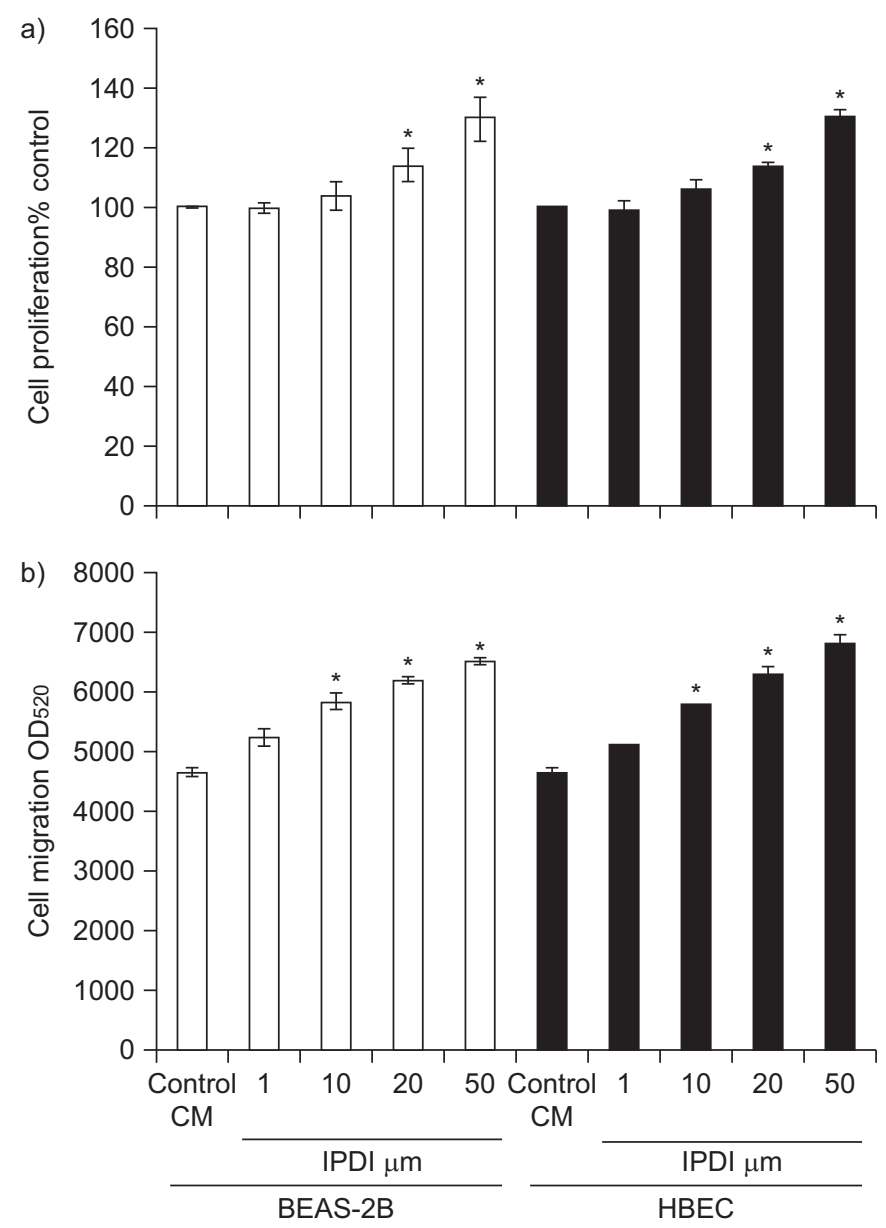

FIGURE 1. The effect of isophorone diisocyanate (IPDI)-treated BEAS-2B cellconditioned medium (CM) (IPDI-BEAS-2B-CM) and IPDI-treated human bronchial epithelial cell (HBEC)-CM (IPDI-HBEC-CM) on the proliferation and migration of bronchial smooth muscle cells (BSMCs). a) IPDI-BEAS-2B-CM and IPDI-HBEC-CM increased BSMC proliferation. b) IPDI-BEAS-2B-CM and IPDI-HBEC-CM enhance the migratory ability of BSMCs. BEAS-2B cells and HBECs $\left(2 \times 10^{6}\right.$ cells per $100-$ $\mathrm{mm}$ dish) were treated with vehicle control or various concentrations of IPDI for $6 \mathrm{~h}$. The medium was replaced with fresh medium, then cells were harvested after $24 \mathrm{~h}$ incubation. The harvested medium was defined as IPDI-BEAS-2B-CM or IPDIHBEC-CM. The effect of IPDI-BEAS-2B-CM and IPDI-HBEC-CM on BSMC proliferation was assessed using Wst-1 Cell Proliferation Reagent after $72 \mathrm{~h}$ incubation. BSMC migration was assessed using the QCM Chemotaxis Cell Migration Assay System. OD530: optical density measured at a wavelength of $520 \mathrm{~nm}$. *: $\mathrm{p}<0.05$

$4 \times 44 \mathrm{k}$ oligo microarray (Agilent Technologies) at $65^{\circ} \mathrm{C}$ for $17 \mathrm{~h}$. After washing and drying by nitrogen-gun blowing, microarrays were scanned by an Agilent microarray scanner (Agilent Technologies), at $535 \mathrm{~nm}$ for Cy3 and $625 \mathrm{~nm}$ for Cy5. Scanned images were analysed with Feature extraction software, version 10.5 (Agilent Technologies), a package used to quantify signal and background intensity for each feature, which substantially normalised the data using the rank-consistency filtering locally weighted scatter-plot smoothing method.

\section{Small interfering RNA knockdown}

BSMCs were transfected with $1 \mu \mathrm{M}$ nontargetting or Rnd3 small interfering (si)RNA pools (Accell; Dharmacon, Lafayette,
CO, USA) in Accell delivery medium (Dharmacon), according to the manufacturer's instructions. Positive controls, Accell GAPDH siRNA and nontargetting Accell siRNA pool were used in the experiments. After $72 \mathrm{~h}$ transfection, the medium was exchanged for complete medium, and the cells treated with a mixture of cytokine and pterostilbene for an additional $15 \mathrm{~h}$. The changes in Rnd3 expression were measured by realtime PCR as described earlier.

\section{Statistical analysis}

Data are presented as mean \pm SD. Statistical comparisons of the results were made using ANOVA. Significant differences $(p<0.05)$ between the means of the two test groups were analysed by Dunnett's test.

\section{RESULTS \\ IPDI-BEAS-2B-CM and IPDI-HBEC-CM increased proliferation and migration of BSMCs}

Increased mass of BSMCs, and decreased distance between BSMCs and bronchial epithelial cells are important features of the remodelled wall in asthmatic airways [5]. These changes in BSMCs can be induced by many factors produced by epithelial cells [5]. We collected IPDI-BEAS-2B-CM and IPDI-HBEC-CM, then assessed the effects of these two conditioned media on the proliferation and migration of BSMCs. As shown in figure 1a, IPDI-BEAS-2B-CM and IPDI-HBEC-CM increased the proliferation of BSMC in a dose-dependent manner after $72 \mathrm{~h}$. Furthermore, IPDI-BEAS-2B-CM and IPDI-HBEC-CM increased the migration of BSMCs in a concentration-dependent manner (fig. 1b).

\section{IPDI-BEAS-2B-CM and IPDI-HBEC-CM increased the expression of inflammatory, adherence and chemotaxis factors}

Increased inflammatory response of BSMCs is a cardinal feature in the development of airway remodelling [15]. We assessed whether IPDI increased the inflammatory response through cross-talk of the epithelium and bronchial smooth muscle. As shown in figure 2a, IPDI-BEAS-2B-CM increased the transcription of inflammatory cytokines, including IL-1 $\beta$, IL-6, IL-8, CXCL2, CXCL3, CXCL5, C-C motif ligant (CCL)5 and intercellular adhesion molecule, as assessed by microarray analysis. These data were confirmed by real-time PCR in IPDIBEAS-2B-CM and IPDI-HBEC-CM-treated BSMCs (fig. 2b). Similarly, IPDI-BEAS-2B-CM and IPDI-HBEC-CM also increased the amount of IL-8, IL-1 $\beta$ and CXCL5 at the protein level (fig. 2c-e).

\section{Involvement of IL-8 in IPDI-BEAS-2B-CM- and IPDI-HBEC- CM-mediated proliferation and migration}

Increased inflammatory response in the epithelium plays an important role in the development of asthma [16, 17]. We consequently assessed whether IPDI increased the production of inflammatory factors in epithelial cells, which in turn enhances the proliferation and migration of BSMCs. We first determined the cytokine profile of BEAS-2B cells after IPDI treatment by cytokine array analysis. The data showed that IPDI only increased the amount of IL-8 (fig. 3b), which was confirmed by ELISA. As shown in figure 3b, IPDI increased the amount of IL-8 in BEAS-2B cells and HBECs in a dosedependent manner. In contrast, IPDI failed to affect IL-6, 

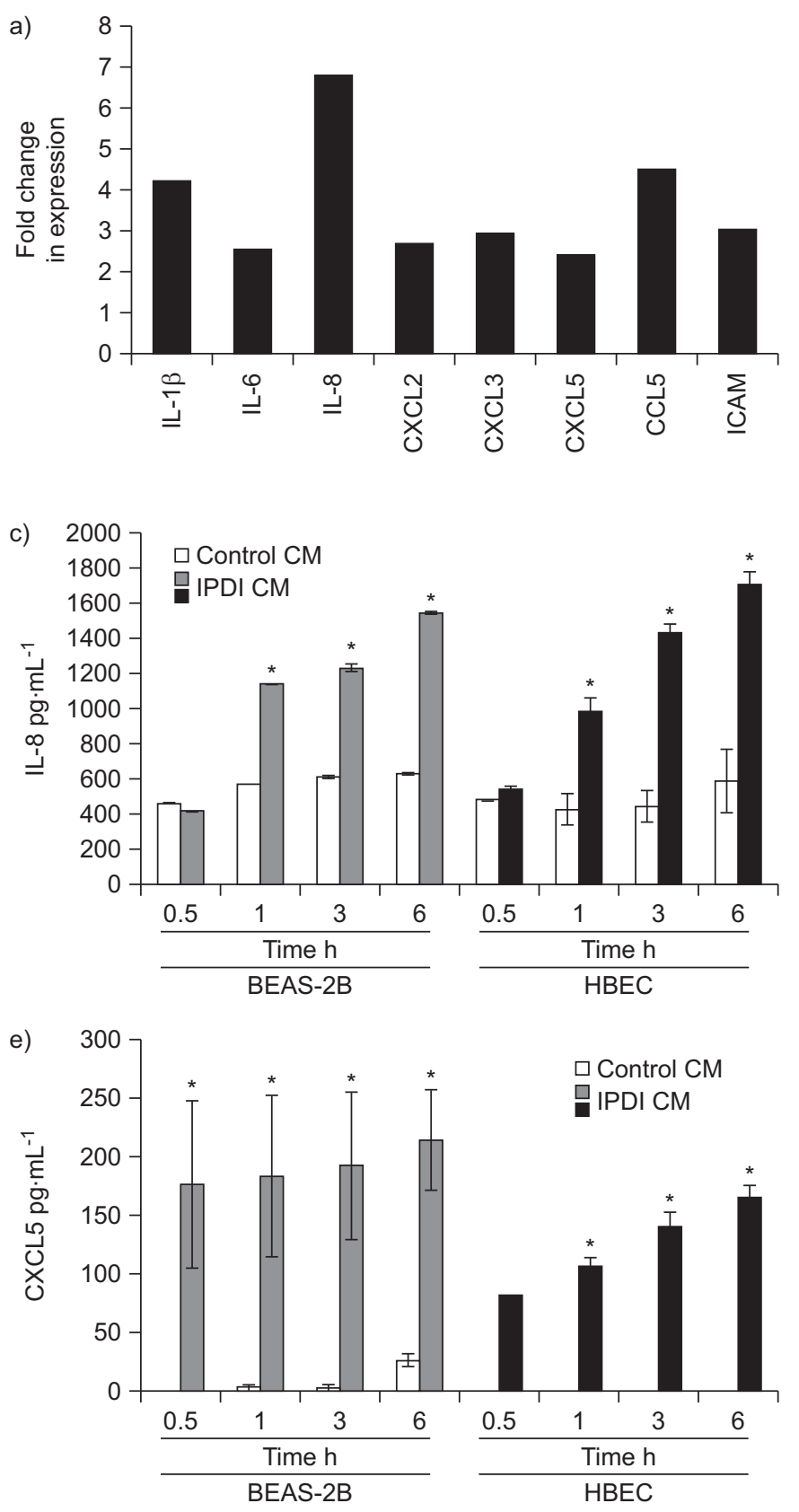

CCL5/RANTES (regulated on activation, normal T-cell expressed and secreted) and CXCL5 levels in BEAS-2B and HBECs (data not shown). We also assessed the effect of rhIL-8 on the proliferation and migration of BSMCs. The results showed that rhIL-8 not only increased BSMC proliferation (fig. 3c), but also enhanced BSMC migration (fig. 3d).

Next, we depleted IPDI-BEAS-2B-CM and IPDI-HBEC-CM of IL-8 to ascertain the role of IL-8 in BSMC proliferation and migration. The successful depletion of IL-8 from IPDI-BEAS2B-CM and IPDI-HBEC-CM was confirmed by IL-8 ELISA (data not shown). As shown in figure 3e, IL-8-depleted IPDIBEAS-2B-CM and IPDI-HBEC-CM were added to BSMCs, effectively reversing the proliferation of BSMCs caused by IPDI-BEAS-2B-CM and IPDI-HBEC-CM. Similarly, increased
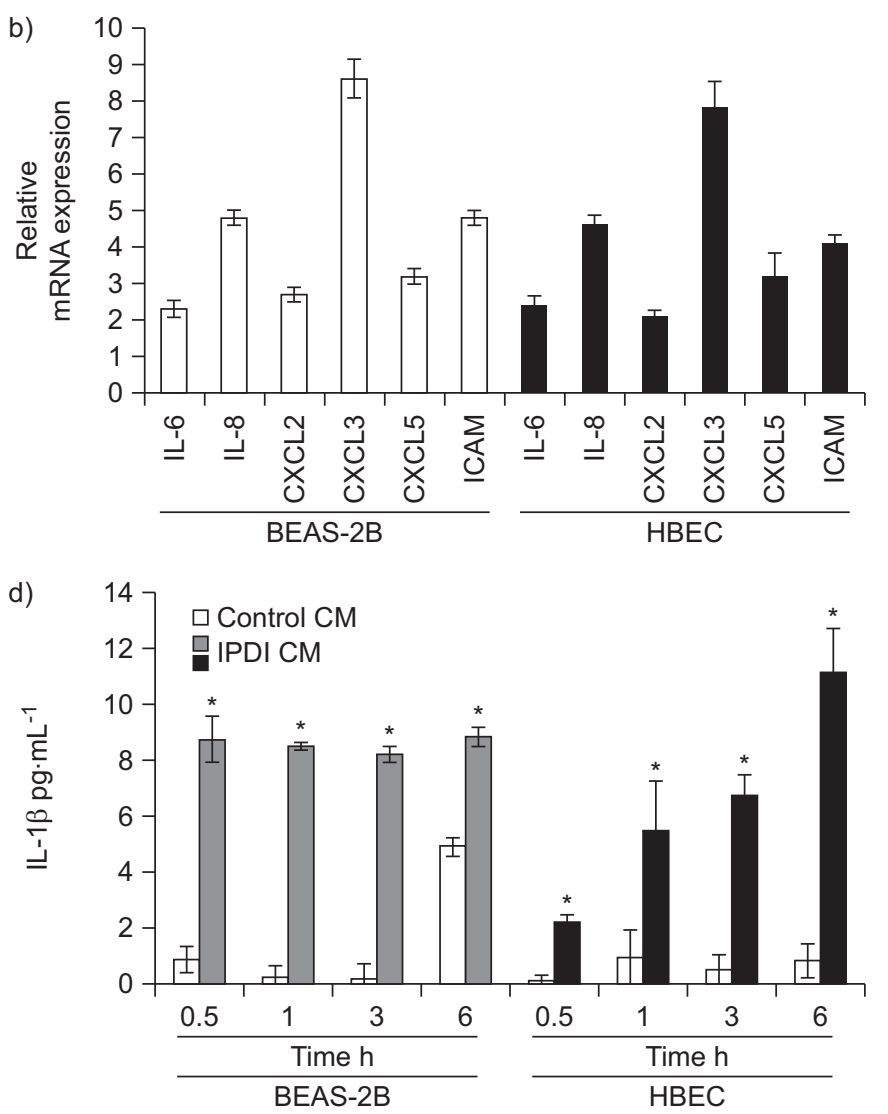

FIGURE 2. $50 \mu \mathrm{M}$ isophorone diisocyanate (IPDI)-treated BEAS-2B cellconditioned medium (CM) (IPDI-BEAS-2B-CM) and IPDI-treated human bronchial epithelial cell (HBEC)-CM (IPDI-HBEC-CM) increased inflammatory and chemotaxis response in bronchial smooth muscle cells (BSMCs). IPDI-BEAS-2B-CM and IPDIHBEC-CM increased the expression of inflammatory factors and chemotaxis at the transcriptional level, as assessed by a) microarray and b) real-time PCR. IPDI-BEAS2B-CM and IPDI-HBEC-CM increased the amounts of C) interleukin (IL)-8, d) IL-1 $\beta$ and e) $C X C$ chemokine ligand $(C X C L) 5$ at the protein level. BSMCs were treated with IPDI-BEAS-2B-CM and IPDI-HBEC-CM for $6 \mathrm{~h}$, and mRNA expression was assessed by a) microarray and b) real-time PCR. $\mathrm{C}-\mathrm{e}$ ) BSMCs were treated with IPDI-BEAS-2B-CM and IPDI-HBEC-CM for the indicated times, and the amounts of various proteins were detected by ELISA. ICAM: intercellular adhesion molecule. $*: p<0.05$.

BSMC migration induced by IPDI-BEAS-2B-CM and IPDIHBEC-CM was terminated upon IL-8 depletion (fig. 3f).

The effects of IPDI-BEAS-2B-CM, IPDI-HBEC-CM and rhIL-8 on the FAK/Rnd3 signalling pathway

FAK is postulated to integrate growth factor, cytokine and integrin signals, and be involved in cell migration. We assessed whether IPDI-BEAS-2B-CM, IPDI-HBEC-CM and rhIL-8 induce BSMC migration via FAK. As shown in figure $4 \mathrm{a}$, IPDI-BEAS-2B-CM, IPDI-HBEC-CM and rhIL-8 increased the phosphorylation of FAK at Tyr397, Tyr576/577 and Tyr925 in BSMCs. However, IPDI-BEAS-2B-CM, IPDI-HBEC-CM and rhIL-8 treatment did not cause any change in the protein levels of total FAK. Exposure of BSMCs to IPDI-BEAS-2B-CM, IPDIHBEC-CM and rhIL-8 increased the active form Src (Tyr416 
a)

\begin{tabular}{|c|c|c|c|c|c|c|c|c|c|c|c|}
\hline A & B & c & D & E & $\mathrm{F}$ & G & $\mathrm{H}$ & I & $\mathrm{J}$ & $\mathrm{K}$ & $\mathrm{L}$ \\
\hline POS & POS & NEG & NEG & EOTAXIN & EOTAXIN-2 & GCSF & GM-CSF & ICAM-1 & IFN- $\gamma$ & I-309 & IL-1 $\alpha$ \\
\hline POS & POS & NEG & NEG & EOTAXIN & EOTAXIN-2 & GCSF & GM-CSF & ICAM-1 & IFN- $\gamma$ & $1-309$ & IL-1 $\alpha$ \\
\hline IL-1 $\beta$ & IL-2 & IL-3 & IL-4 & IL-6 & IL-6sR & IL-7 & IL-8 & IL-10 & IL-11 & IL-12p40 & IL-12p70 \\
\hline IL-1 $\beta$ & IL-2 & IL-3 & IL-4 & IL-6 & IL-6sR & IL-7 & IL-8 & IL-10 & IL-11 & IL-12p40 & IL-12p70 \\
\hline IL-13 & IL-15 & IL-16 & IL-17 & IP-10 & MCP-1 & MCP-2 & M-CSF & MIG & MIP-1 $\alpha$ & MIP-1 $\beta$ & 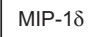 \\
\hline IL-13 & IL-15 & IL-16 & IL-17 & IP-10 & MCP-1 & MCP-2 & M-CSF & MIG & MIP-1 $\alpha$ & MIP-1 $\beta$ & MIP-1 $\delta$ \\
\hline RANTES & TGF- $\beta 1$ & TNF- $\alpha$ & TNF- $\beta$ & sTNF RI & sTNF RII & PDGF-BB & TIMP-2 & BLANK & BLANK & NEG & POS \\
\hline RANTES & TGF- $\beta 1$ & TNF- $\alpha$ & TNF- $\beta$ & sTNF RI & sTNF RII & PDGF-BB & TIMP-2 & BLANK & BLANK & NEG & POS \\
\hline
\end{tabular}
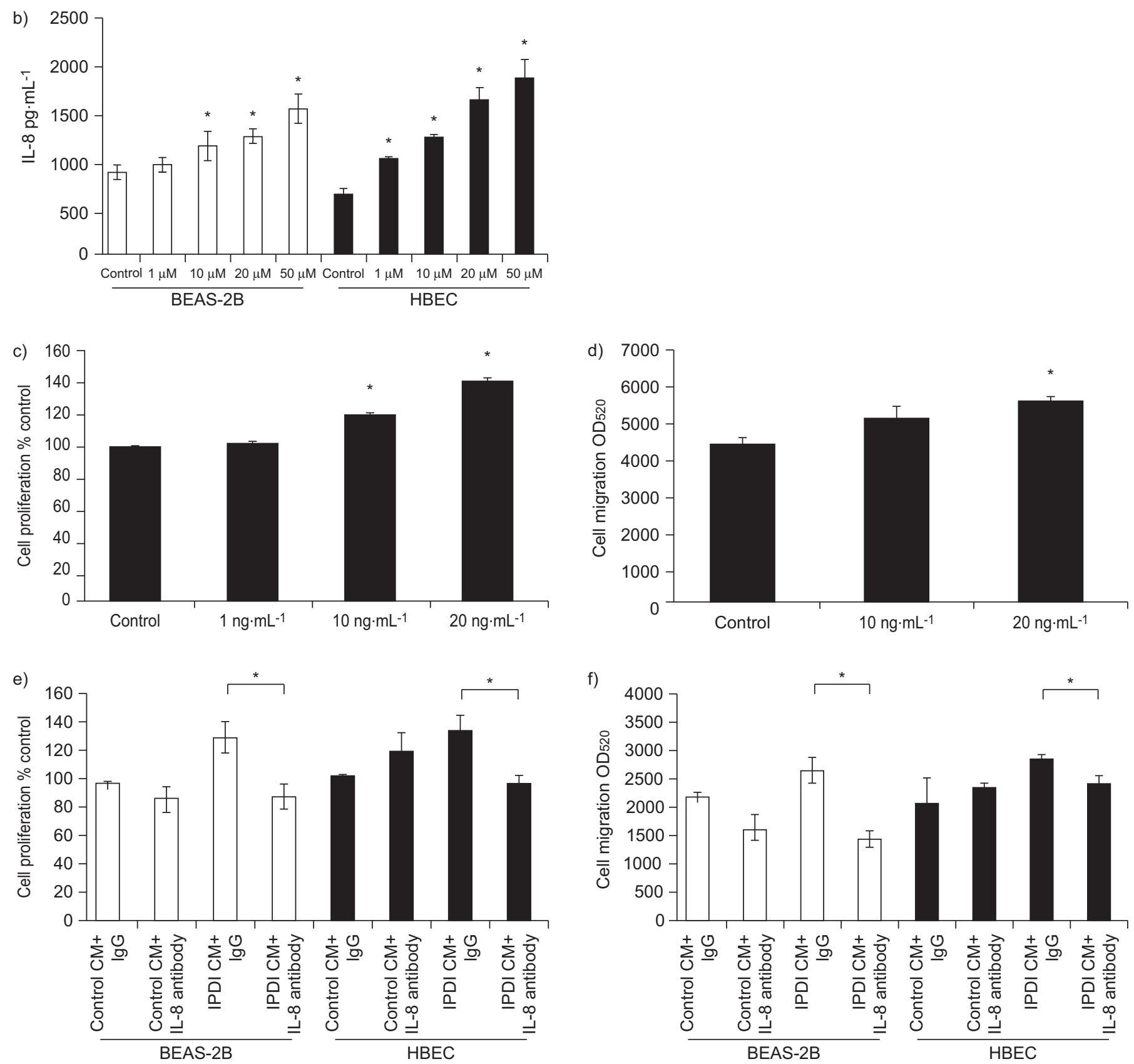
FIGURE 3. Interleukin (IL)-8 is involved in bronchial smooth muscle cell (BSMC) proliferation and migration induced by isophorone diisocyanate (IPDI)-treated BEAS-2B cell-conditioned medium (CM) (IPDI-BEAS-2B-CM) and IPDI-treated human bronchial epithelial cell (HBEC)-CM (IPDI-HBEC-CM). IPDI increased the amount of IL-8 in BEAS$2 \mathrm{~B}$ cells, as assessed by a) cytokine array analysis and b) ELISA. The addition of recombinant human IL-8 increased BSMC c) proliferation and d) migration. Depletion of IL-8 from IPDI-BEAS-2B-CM and IPDI-HBEC-CM decreased BSMC e) proliferation and f) migration induced by IPDI-BEAS-2B-CM and IPDI-HBEC-CM. BEAS-2B cells and HBECs were treated with IPDI $(50 \mu \mathrm{M})$ for $6 \mathrm{~h}$. The supernatants were collected and the IL-8 levels of IPDI-BEAS-2B-CM and IPDI-HBEC-CM assessed by cytokine array analysis and ELISA. POS: positive control; NEG: negative control; GCSF: granulocyte colony-stimulating factor; GM-CSF: granulocyte-macrophage colony-stimulating factor; ICAM: intercellular adhesion molecule; IFN: interferon; sR: soluble receptor; IP: IFN- $\gamma$-induced protein; MCP: monocyte chemotactic protein; M-CSF: macrophage colonystimulating factor; MIG: monokine induced by IFN- $\gamma$; MIP: macrophage inflammatory protein; TGF: transforming growth factor; TNF: tumour necrosis factor; sTNFR: soluble TNF receptor; PDGF: platelet-derived growth factor; TIMP: tissue inhibitor of metalloproteases; OD520: optical density measured at a wavelength of $520 \mathrm{~nm}$. ${ }^{*}: \mathrm{p}<0.05$.

phosphorylation) and decreased the inactive form Src (Tyr527). In addition, the association of FAK and Src increased in a timedependent manner in IPDI-BEAS-2B-CM-, IPDI-HBEC-CMand rhIL-8-treated BSMC, as determined by immunoprecipitation assay (fig. 4b). Similar responses were observed for the phosphorylated forms of two other downstream targets of FAK, ERK1/2 and AKT (fig. 4a).

Because Rnd3/RhoE is a target of MEK-ERK1/2 signalling [12], we assessed the expression of Rnd3 in BSMCs. As shown in figure $4 \mathrm{c}$ and $\mathrm{d}$, IPDI-BEAS-2B-CM, IPDI-HBEC-CM and rhIL-8 increased the amount of Rnd3 at both the mRNA and protein levels in BSMCs.

\section{The role of FAK and ERK in BSMC proliferation and migration}

In order to investigate the factors upstream of Rnd3, we assessed the roles of FAK and ERK1/2 on the expression of Rnd3 using specific chemical inhibitors. BSMCs were pretreated with FAK inhibitor 14 (inhibitor of FAK) and PD98059 (inhibitor of ERK1/2), after which the cells were exposed to IPDI-BEAS-2B-CM, IPDI-HBEC-CM and rhIL-8. The effect of FAK and ERK inhibitors on the expression of Rnd3, cell migration and proliferation was then examined. Pre-treatment of BSMCs with FAK inhibitors reduced the phosphorylation of FAK induced by IPDI-BEAS-2B-CM, IPDI-HBEC-CM or rhIL-8 (fig. 5a). The specific inhibitor of FAK halted the ERK1/2 phosphorylation and Rnd3 upregulation in BSMCs after IPDI-BEAS-2B-CM, IPDI-HBEC-CM and rhIL-8 treatment (fig. 5a). Cell proliferation and migration also ceased in BSMCs after IPDI-BEAS-2B-CM, IPDI-HBEC-CM and rhIL-8 treatment (fig. 5b-e).

Specific inhibition of ERK1/2 activation by PD98059 decreased IPDI-BEAS-2B-CM-, IPDI-HBEC-CM- and rhIL-8-induced Rnd3 upregulation in BSMCs (fig. 6a). Similarly to FAK inhibition, PD98059 also decreased the effect of IPDI-BEAS2B-CM, IPDI-HBEC-CM and rhIL-8 on BSMC proliferation and migration (fig. 6b-e).

The role of Rnd3 in BSMC migration induced by IPDI-BEAS2B-CM, IPDI-HBEC-CM and rhIL-8

To confirm the central role of Rnd3 in BSMC migration induced by IPDI-BEAS-2B-CM, IPDI-HBEC-CM and rhIL-8, we transfected BSMC with Rnd3 siRNA. As shown in figure 7a, Rnd3 siRNA reduced Rnd3 expression by $\sim 90 \%$, in comparison with control siRNA transfection. Specific knockdown of Rnd3 expression decreased the effect of IPDI-BEAS-2B-CM, IPDIHBEC-CM and rhIL-8 on migration in BSMCs, but failed to affect BSMC proliferation (fig. $7 \mathrm{~b}-\mathrm{e}$ ). These results suggest that Rnd3 may play a key role in IPDI-mediated BSMC migration.

\section{DISCUSSION}

This study is the first to investigate the interaction of airway epithelium and smooth muscle after exposure to the environmental chemical agent IPDI. IPDI caused immortalised, nontumorigenic human bronchial epithelial cells (cell line BEAS-2B) and primary normal HBECs to produce IL-8, which increased the proliferation and migration of primary normal human BSMCs. In addition, IPDI-BEAS-2B-CM, IPDI-HBEC$\mathrm{CM}$ and rhIL-8 increased FAK activation, subsequently increasing ERK1/2 and AKT activation in BSMCs. In short, blocking the upstream molecule FAK or downstream factor ERK1/2 activation using specific inhibitors effectively reverses their inductive effect on BSMC proliferation and migration. Furthermore, inhibition of Rnd3 by siRNA also decreased IPDI-BEAS-2B-CM-, IPDI-HBEC-CM- and rhIL-8-mediated BSMC migration. These data suggest that FAK, ERK1/2 and Rnd3 play a critical role in IPDI-mediated asthma (fig. 8).

CXCL8/IL-8, a pro-inflammatory CXC chemokine, is secreted by a variety of human bronchial epithelial cells or BSMCs exposed to pro-inflammatory cytokines, such as IL-1 and tumour necrosis factor- $\alpha$, and promotes an inflammatory response [17, 18]. IL-8 has been reported to trigger calcium release, contraction and migration in BSMCs through functional CXC chemokine receptor (CXCR)1 and CXCR2 [18, 19]. Another isocyanate, toluene diisocyanate, has been found to increase bronchial epithelial cells production of IL-8, which in turn recruits neutrophils, resulting in an inflammatory response [20, 21]. However, the role of IL-8 on IPDI-related asthma remains unknown. We found that IPDI increases BEAS-2B cell and HBEC secretion of IL-8, which in turn increases the proliferation and migration of BSMCs. However, depleting IL-8 from IPDI-BEAS-2B-CM and IPDI-HBEC-CM reversed that. Moreover, treatment of BSMC with IPDI also increased their proliferation and migration. This is an important correlation to our finding on the clinical significance of elevated IL-8 levels in IPDI-mediated asthma.

FAK, a nonreceptor tyrosine kinase, has been shown to be a critical mediator of cell proliferation, survival and migration in a variety of cell types [22]. Many studies have reported that activation of FAK is strongly associated with vascular smooth muscle cell growth and migration, whereas loss of FAK activation inhibited smooth muscle cell proliferation and migration [22, 23]. Activated FAK can associate with Src to form a complex, which relieves inactivated phosphorylation at Tyr527 and promotes activated autophosphorylation at Tyr416, resulting in Src activation. Activated Src has been shown to phosphorylate FAK at multiple tyrosine residues, subsequently activating AKT and ERK1/2, and promoting cell proliferation and migration. Although LIN et al. [22] reported that inhibition 
a)

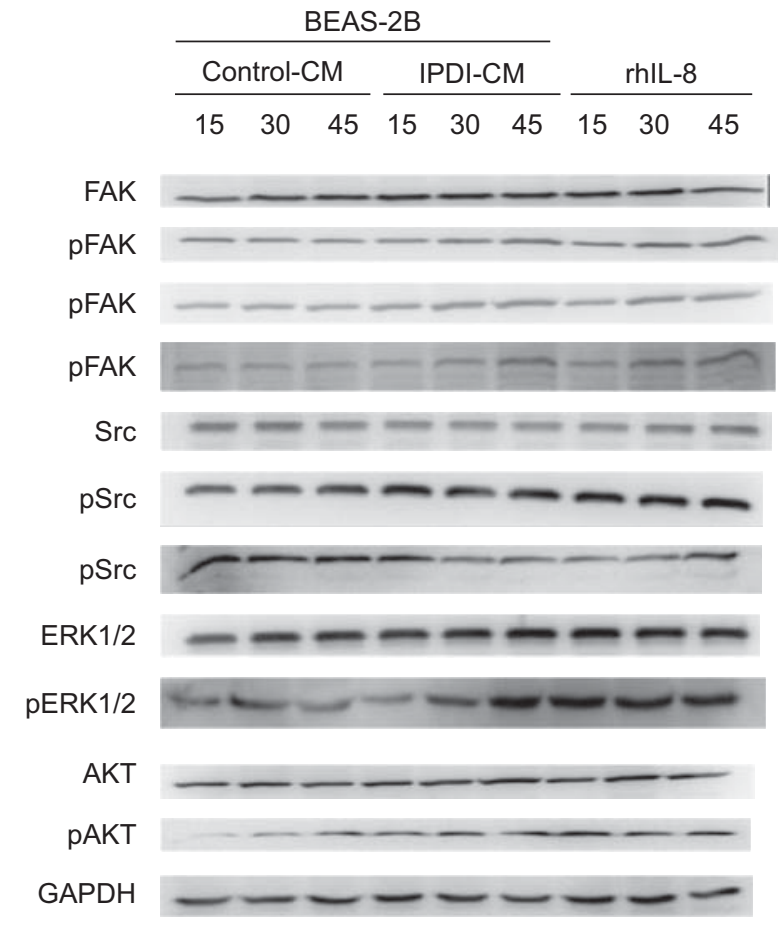

HBEC

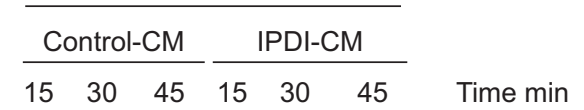
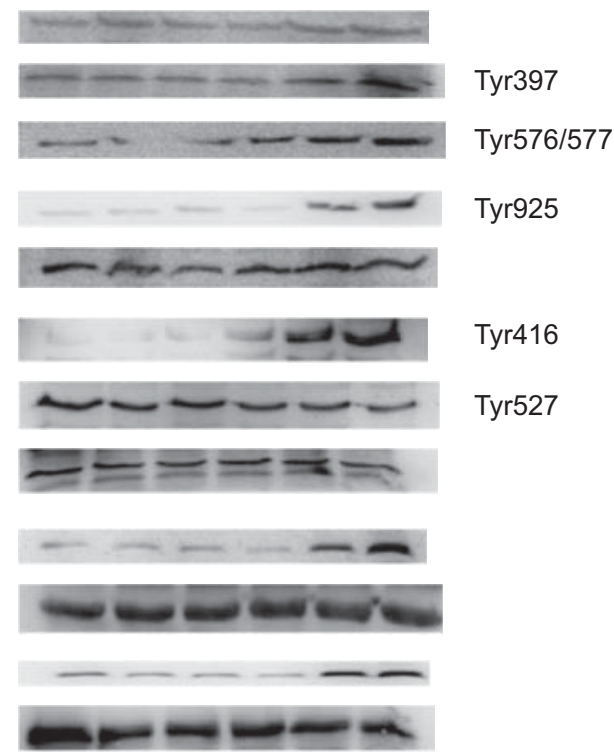

b)

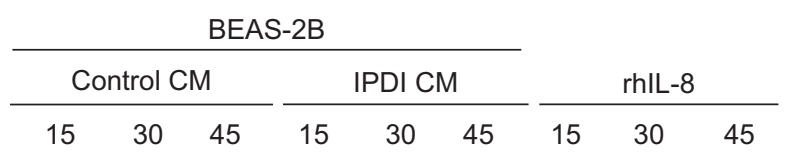

IP: FAK

IB: SrC

IP: FAK

IB: FAK
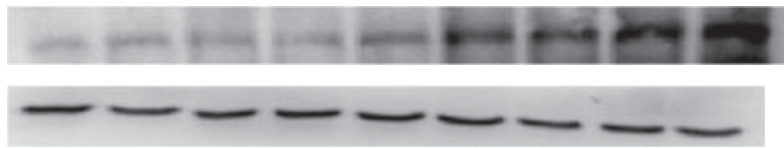

HBEC

\begin{tabular}{|c|c|}
\hline Control CM & IPDI CM \\
\hline $\begin{array}{lll}15 & 30 & 45\end{array}$ & $30 \quad 45$ \\
\hline
\end{tabular}

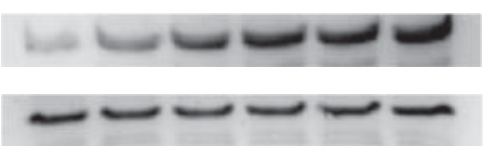

c)

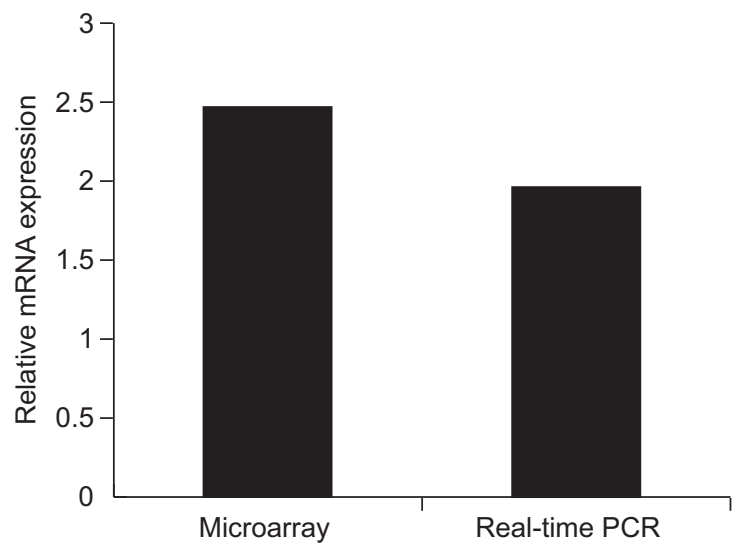

d)
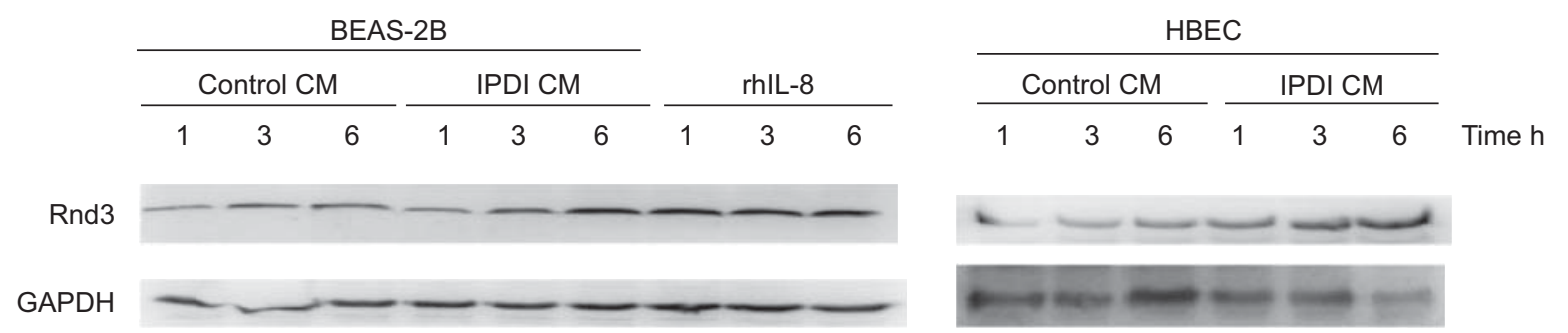
FIGURE 4. $50 \mu \mathrm{M}$ isophorone diisocyanate (IPDI)-treated BEAS-2B cell-conditioned medium (CM) (IPDI-BEAS-2B-CM), IPDI-treated human bronchial epithelial cell (HBEC)-CM (IPDI-HBEC-CM) and recombinant human interleukin (rhIL)-8 activate focal adhesion kinase (FAK) signalling. a) IPDI-BEAS-2B-CM, IPDI-HBEC-CM and rhIL-8 increased the phosphorylation of FAK, Src, extracellular signal-regulated kinase (ERK)1/2 and AKT in bronchial smooth muscle cells (BSMCs). b) IPDI-BEAS-2B-CM, IPDIHBEC-CM and rhIL-8 enhanced the interaction of FAK and Src. IPDI-BEAS-2B-CM, IPDI-HBEC-CM and rhIL-8 increased the amount of Rnd3 at both the c) mRNA and d) protein levels. BSMCs were treated with IPDI-BEAS-2B-CM, IPDI-HBEC-CM and rhlL-8 $\left(20 \mathrm{ng} \cdot \mathrm{mL}^{-1}\right)$ for the indicated times. Phosphorylated ( $\mathrm{p}$ ) and unphosphorylated proteins were assessed by immunoblotting (IB), and the interaction of FAK and Src detected by immunoprecipitation (IP). The amount of Rnd3 mRNA was assayed by microarray analysis and real-time PCR. GAPDH: glyceraldehyde phosphate dehydrogenase.

of FAK with antisense oligodeoxynucleotides inhibits human pulmonary artery smooth muscle cells proliferation, resulting in cell apoptosis, the effect of FAK on bronchial smooth muscle remained unknown. In our study, we found that IPDI-BEAS-2BCM, IPDI-HBEC-CM and rhIL-8 increased the phosphorylation of FAK at three different tyrosine sites. and enhanced the

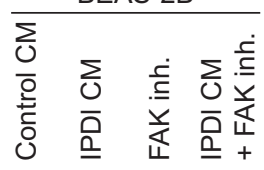

pFAK

pERK $1 / 2$

Rnd3

GAPDH

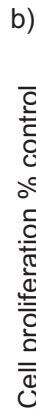

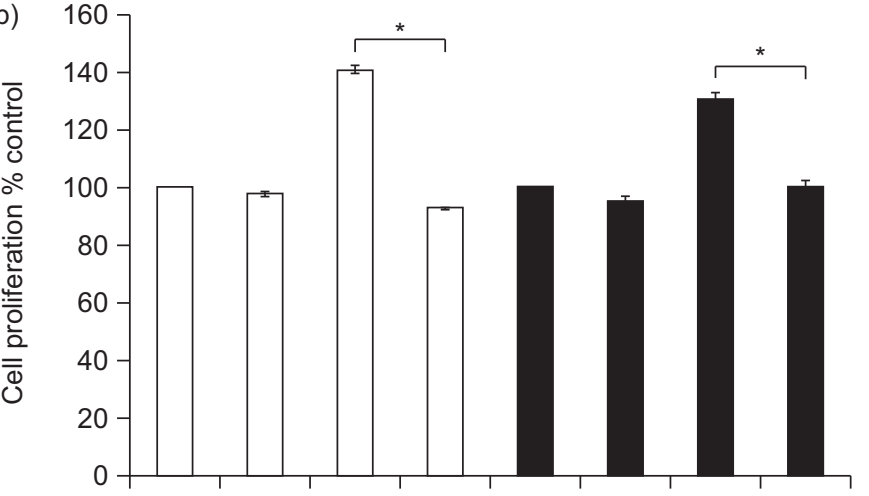

d)

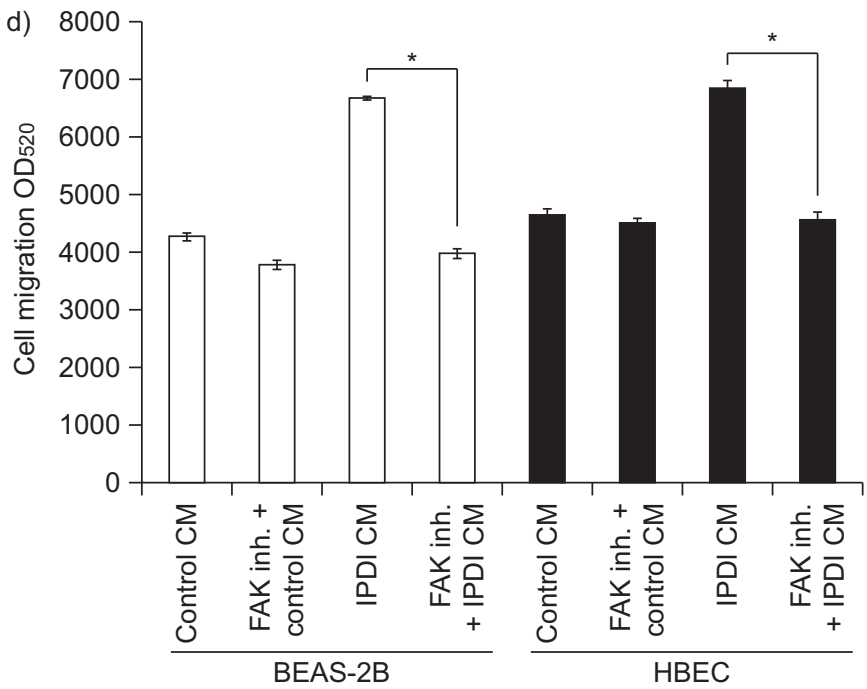

HBEC
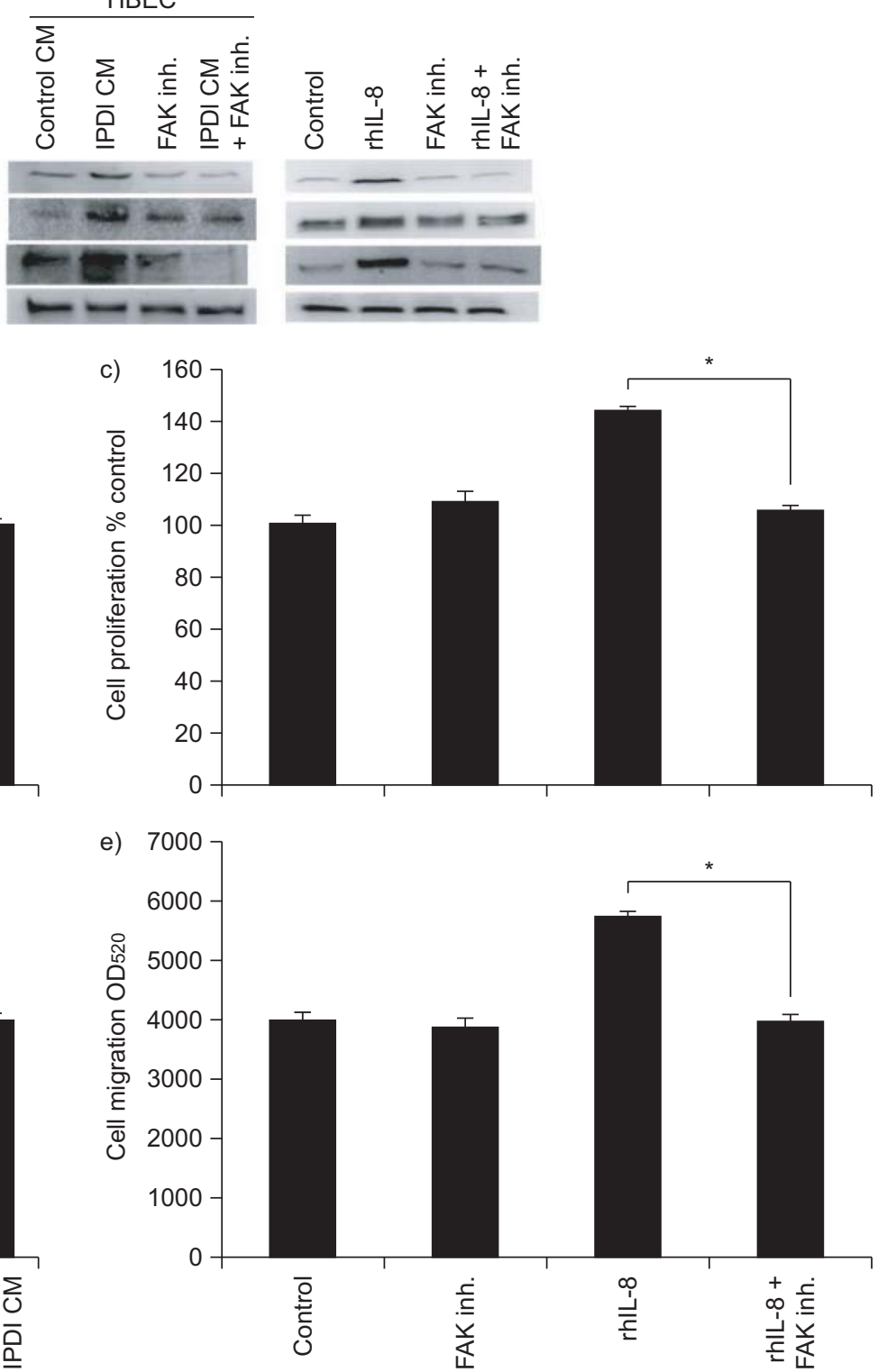

FIGURE 5. The role of focal adhesion kinase (FAK) on bronchial smooth muscle cell (BSMC) proliferation and migration. a) The effects of FAK inhibitor (inh.) on the phosphorylation ( $p$ ) of FAK and extracellular signal-regulated kinase (ERK)1/2, and Rnd3 expression. The effect of FAK inh. on BSMC proliferation caused by b) isophorone diisocyanate (IPDI)-treated BEAS-2B cell-conditioned medium (CM) (IPDI-BEAS-2B-CM) and IPDI-treated human bronchial epithelial cell (HBEC)-CM (IPDI-HBEC-CM), and c) recombinant human interleukin (rhIL)-8. The effect of FAK inh. on BSMC migration induced by d) IPDI-BEAS-2B-CM and IPDI-HBEC-CM, and e) rhIL-8. BSMCs were pretreated with FAK inh. for $1 \mathrm{~h}$ then exposed to IPDI-BEAS-2B-CM, IPDI-HBEC-CM and rhIL-8 (45 min for ERK phosphorylation, $3 \mathrm{~h}$ for Rnd3 expression, $72 \mathrm{~h}$ for proliferation assay and $24 \mathrm{~h}$ for migration assay). GAPDH: glyceraldehyde phosphate dehydrogenase; OD520: optical density measured at a wavelength of $520 \mathrm{~nm}$. *: $\mathrm{p}<0.05$. 
a)
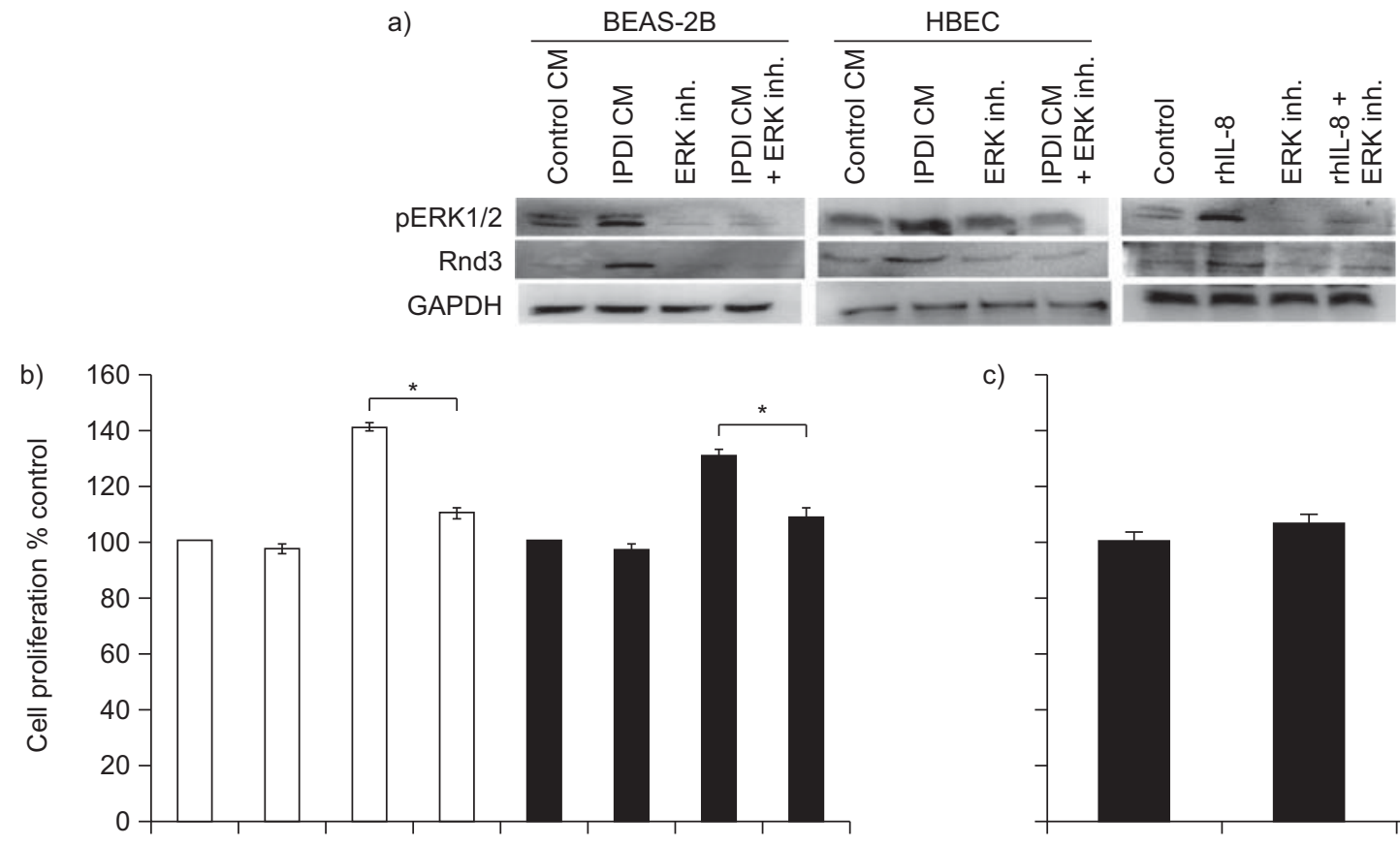

c)

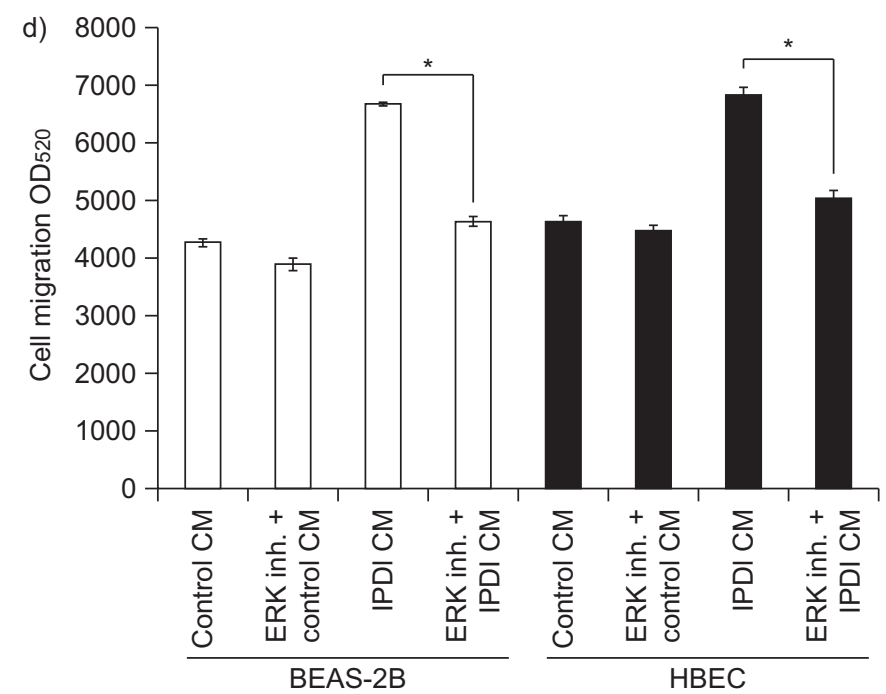

e)
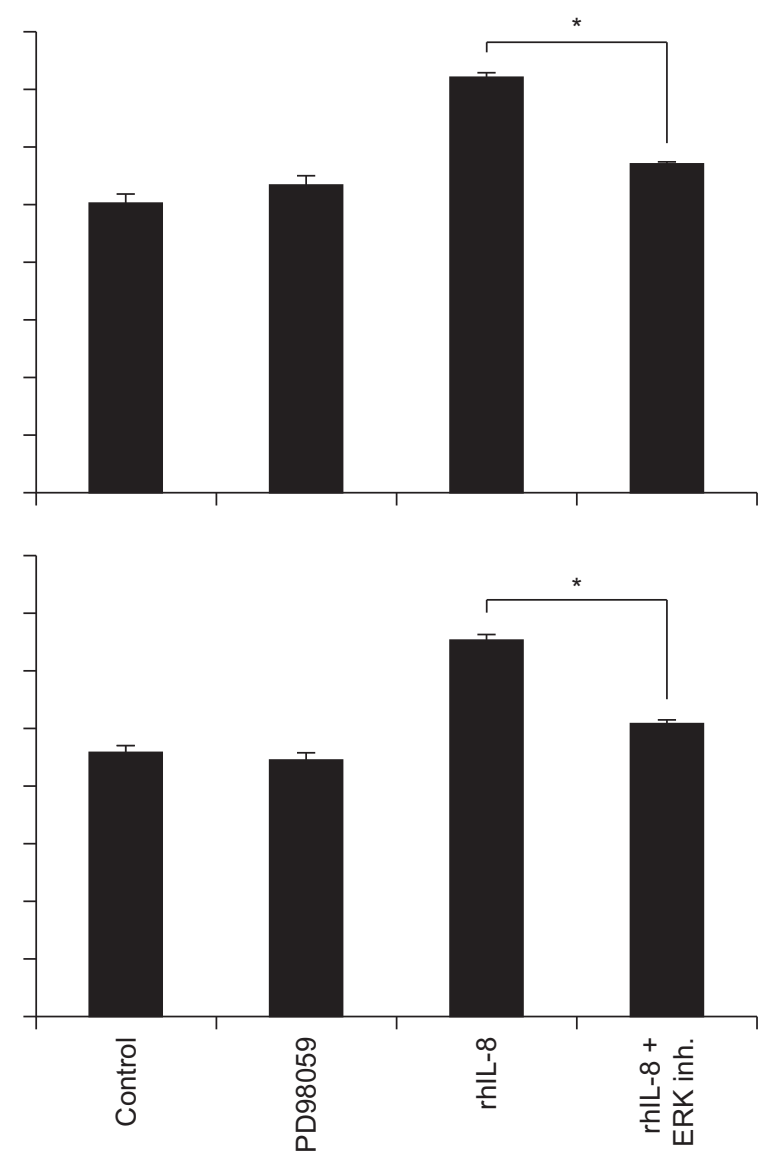

FIGURE 6. The role of extracellular signal-regulated kinase (ERK) 1/2 on bronchial smooth muscle cell (BSMC) proliferation and migration. a) The effects of ERK inhibitor (inh.) on the phosphorylation (p) of ERK1/2 and Rnd3 expression. The effect of ERK inh. on BSMC proliferation induced by b) isophorone diisocyanate (IPDI)-treated BEAS-2B cell-conditioned medium (CM) (IPDI-BEAS-2B-CM) and IPDI-treated human bronchial epithelial cell (HBEC)-CM (IPDI-HBEC-CM), and c) recombinant human interleukin (rhIL)-8. The effect of ERK inh. on BSMC migration caused by d) IPDI-BEAS-2B-CM and IPDI-HBEC-CM, and e) rhIL-8. BSMCs were pre-treated with PD98059 (20 $\mu$ M) for $1 \mathrm{~h}$ then exposed to IPDI-BEAS-2B-CM, IPDI-HBEC-CM and rhIL-8 (45 min for ERK phosphorylation, $3 \mathrm{~h}$ for Rnd3 expression, $72 \mathrm{~h}$ for proliferation assay and $24 \mathrm{~h}$ for migration assay). GAPDH: glyceraldehyde phosphate dehydrogenase; OD520: optical density measured at a wavelength of $520 \mathrm{~nm}$. *: $\mathrm{p}<0.05$

interaction of FAK and Src. In addition, exposure of BSMCs to IPDI-BEAS-2B-CM, IPDI-HBEC-CM and rhIL-8 also activated $A K T$ and ERK1/2. Inhibition of FAK activation decreased ERK1/2 activation, suggesting that FAK is the upstream molecule of ERK1/2. In addition, selective inhibition of FAK and ERK1/2 by chemical inhibitors also decreased the effects of IPDI-BEAS-2B-CM, IPDI-HBEC-CM or rhIL-8 on BSMC proliferation and migration, suggesting that FAK/ERK1/2 plays a crucial role in IPDI-BEAS-2B-CM-, IPDI-HBEC-CM- and rhIL-8mediated BSMC changes.

Rnd3/RhoE, an atypical Rho family protein, exhibits a regulatory role in many cellular processes, such as cytoskeleton formation, cell survival, apoptosis, cell cycle progression and differentiation [23, 24]. Unlike typical Rho proteins, with activity dependent on the guanosine tri- or diphosphate-binding states, the activation of RhoE is controlled by transcription or posttranscriptional modification [25, 26]. However, the function of endogenous Rnd3 in BSMCs remains unknown. Recent studies indicate that endogenous Rnd3 is associated with Rho-associated protein kinase-mediated apoptosis and myoblast alignment [14]. In this study, we have shown that treatment of BSMCs with IPDI-BEAS-2B-CM, IPDI-HBEC-CM or rhIL-8 resulted in increased Rnd3 expression at both the transcriptional and translation levels. Inhibition of FAK and ERK1/2 decreased 
a)
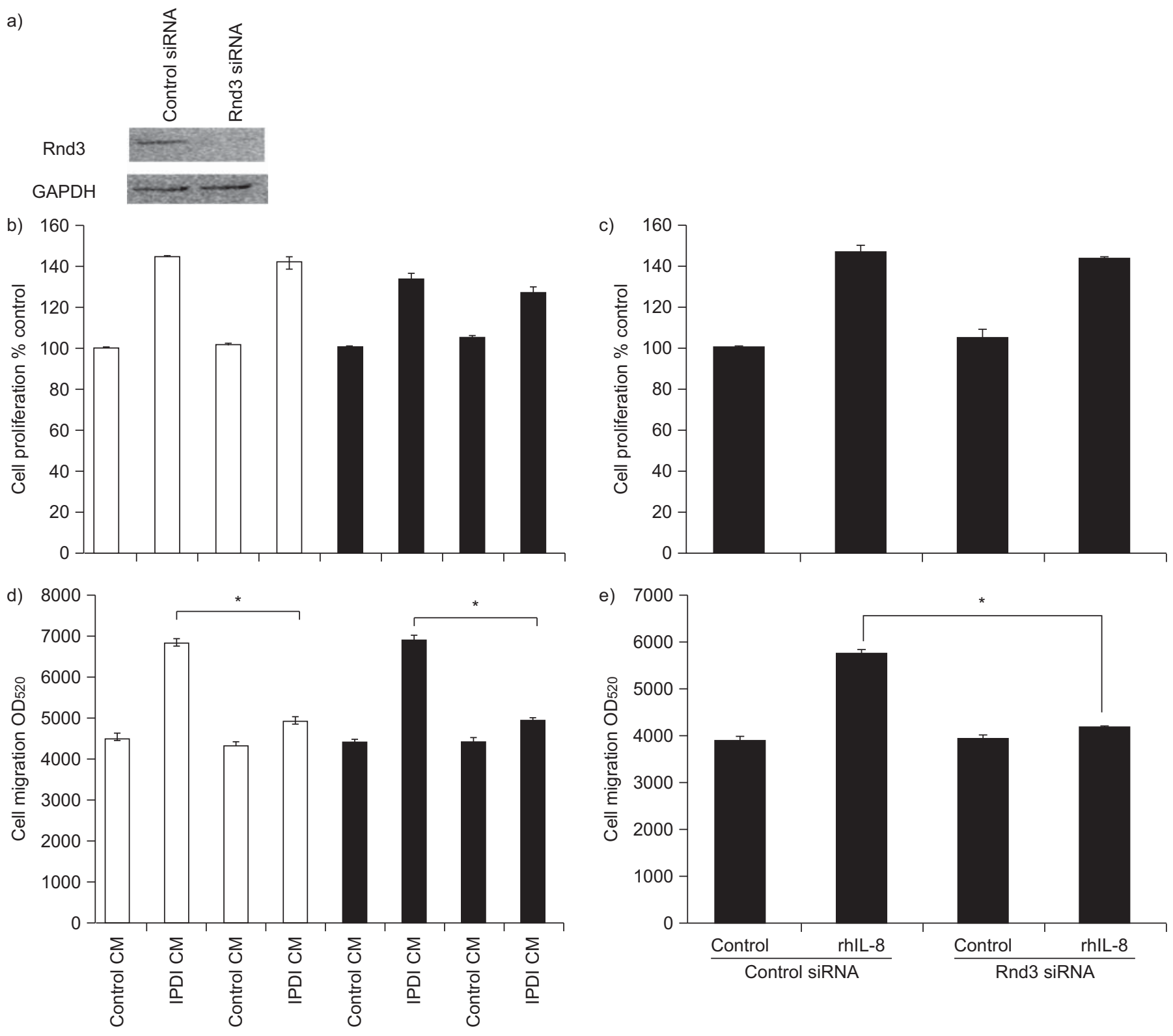

Control siRNA Rnd3 siRNA Control siRNA Rnd3 siRNA BEAS-2B

HBEC

FIGURE 7. The role of Rnd 3 in bronchial smooth muscle cell (BSMC) proliferation and migration. a) Rnd3 was knocked down using small interfering (si)RNA. Rnd3 inhibition did not affect either the inductive effect of b) $50 \mu \mathrm{M}$ isophorone diisocyanate (IPDI)-treated BEAS-2B cell-conditioned medium (CM) (IPDI-BEAS-2B-CM) or IPDItreated human bronchial epithelial cell (HBEC)-CM (IPDI-HBEC-CM), or c) $20 \mathrm{ng} \cdot \mathrm{mL}^{-1}$ recombinant human interleukin (rhIL)-8 on BSMC proliferation. Rnd3 inhibition decreased d) IPDI-BEAS-2B-CM- and IPDI-HBEC-CM-, and e) rhIL-8-mediated BSMC migration. GAPDH: glyceraldehyde phosphate dehydrogenase. *: p<0.05.

the upregulation of Rnd3 by IPDI-BEAS-2B-CM, IPDI-HBEC$\mathrm{CM}$ and rhIL-8, suggesting that FAK/ERK1/2 is the upstream event of Rnd3. In addition, knockdown Rnd3 by siRNA decreased IPDI-BEAS-2B-CM, IPDI-HBEC-CM and rhIL-8mediated BSMC migration, indicating that Rnd3 participates in the migration of BSMC. The regulatory role of Rnd3 on BSMC migration provides a critical new function for Rnds involved in airway remodelling.

Taken together, our findings indicate that conditioned media from IPDI-treated epithelial cells stimulate bronchial epithelial cell proliferation and migration. IL- 8 in the conditioned medium results in an enhanced effect on BSMC growth and movement, while FAK, ERK and Rnd3 have been found to be responsible for BSMC migration. In light of this finding, inhibition of IL-8 signalling is an attractive therapeutic target for IPDI-induced occupational asthma.

\section{SUPPORT STATEMENT}

This study was supported by grants from the Center of Excellence for Environmental Medicine, Kaohsiung Medical University (KMU-EM98-4), Kaohsiung Medical University Research Foundation (KMUQ099002) and Kaohsiung Medical University Hospital (KMUH98-8R21). 


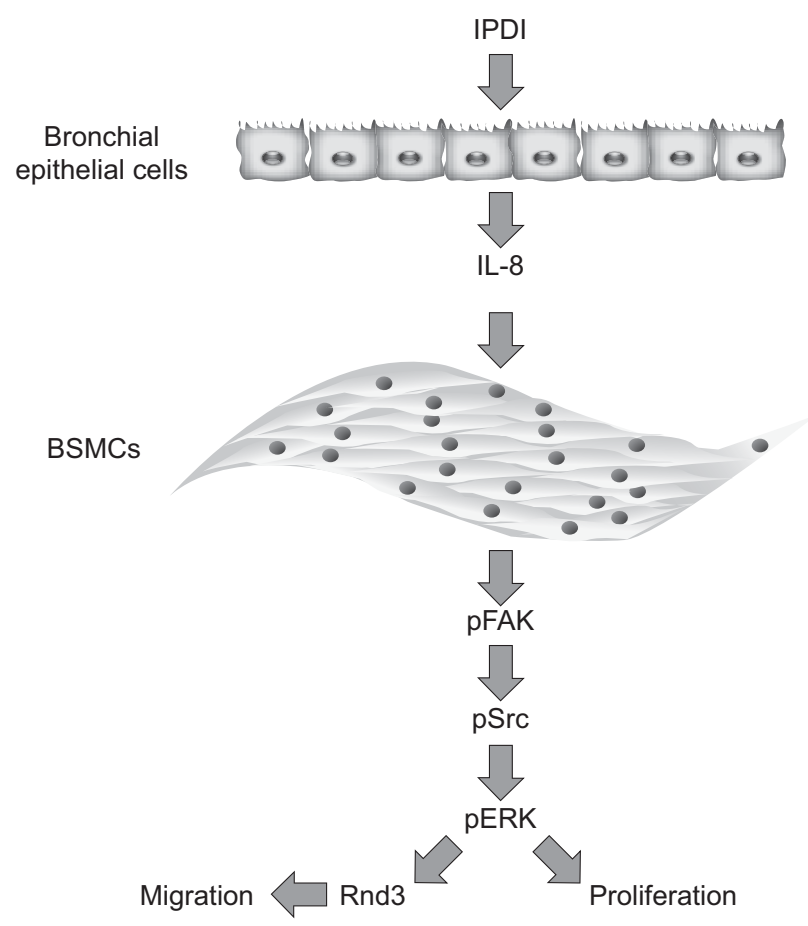

FIGURE 8. The molecular mechanism of isophorone diisocyanate (IPDI)induced asthma. IPDI caused bronchial epithelial cells to produce interleukin (IL)-8, which increased the proliferation and migration of bronchial smooth muscle cells (BSMCs). IL-8 in the conditioned medium results in an enhanced effect on BSMC growth and movement, while focal adhesion kinase (FAK), extracellular signalregulated kinase (ERK) and Rnd3 has been found to be responsible for BSMC migration. p: phosphorylated.

\section{STATEMENT OF INTEREST}

None declared.

\section{REFERENCES}

1 Farraj AK, Boykin E, Haykal-Coates N, et al. Th2 cytokines in skin draining lymph nodes and serum IgE do not predict airway hypersensitivity to intranasal isocyanate exposure in mice. Toxicol Sci 2007; 100: 99-108.

2 Clarke CW, Aldons PM. Isophorone diisocyanate induced respiratory disease (IPDI). Aust N Z J Med 1981; 11: 290-292.

3 Labrecque M, Khemici E, Cartier A, et al. Impairment in workers with isocyanate-induced occupational asthma and removed from exposure in the province of Québec between 1985 and 2002. J Occup Environ Med 2006; 48: 1093-1098.

4 Lange RW, Lantz RC, Stolz DB, et al. Toluene diisocyanate colocalizes with tubulin on cilia of differentiated human airway epithelial cells. Toxicol Sci 1999; 50: 64-71.

5 Bergeron C, Al-Ramli W, Hamid Q. Remodeling in asthma. Proc Am Thorac Soc 2009; 6: 301-305.

6 Benayoun L, Druilhe A, Dombret MC, et al. Airway structural alterations selectively associated with severe asthma. Am J Respir Crit Care Med 2003; 167: 1360-1368.

7 Cohen-Hillel E, Yron I, Meshel T, et al. CXCL8-induced FAK phosphorylation via CXCR1 and CXCR2: cytoskeleton- and integrin-related mechanisms converge with FAK regulatory pathways in a receptor-specific manner. Cytokine 2006; 33: 1-16.

8 Bond M, Sala-Newby GB, Newby AC. Focal adhesion kinase (FAK)-dependent regulation of S-phase kinase-associated protein-2 (Skp-2) stability. A novel mechanism regulating smooth muscle cell proliferation. J Biol Chem 2004; 279: 37304-37310.

9 Cohen LA, Guan JL. Mechanisms of focal adhesion kinase regulation. Curr Cancer Drug Targets 2005; 5: 629-643.

10 Fiegen D, Blumenstein L, Stege P, et al. Crystal structure of Rnd3/ RhoE: functional implications. FEBS Lett 2002; 525: 100-104.

11 Klein RM, Aplin AE. Rnd3 regulation of the actin cytoskeleton promotes melanoma migration and invasive outgrowth in three dimensions. Cancer Res 2009; 69: 2224-2233.

12 Klein RM, Spofford LS, Abel EV, et al. B-RAF regulation of Rnd3 participates in actin cytoskeletal and focal adhesion organization. Mol Biol Cell 2008; 19: 498-508.

13 Poch E, Miñambres R, Mocholí E, et al. RhoE interferes with $\mathrm{Rb}$ inactivation and regulates the proliferation and survival of the U87 human glioblastoma cell line. Exp Cell Res 2007; 313: 719-31.

14 Fortier M, Comunale F, Kucharczak J, et al. RhoE controls myoblast alignment prior fusion through RhoA and ROCK. Cell Death Differ 2008; 15: 1221-1231.

15 Tliba O, Panettieri RA Jr. Regulation of inflammation by airway smooth muscle. Curr Allergy Asthma Rep 2008; 8: 262-268.

16 Holgate ST. Epithelium dysfunction in asthma. J Allergy Clin Immunol 2007; 120: 1233-1244.

17 Takeda N, Sumi Y, Préfontaine D, et al. Epithelium-derived chemokines induce airway smooth muscle cell migration. Clin Exp Allergy 2009; 39: 1018-1026.

18 Govindaraju V, Michoud MC, Al-Chalabi M, et al. Interleukin-8: novel roles in human airway smooth muscle cell contraction and migration. Am J Physiol Cell Physiol 2006; 291: C957-C965.

19 John AE, Zhu YM, Brightling CE, et al. Human airway smooth muscle cells from asthmatic individuals have CXCL8 hypersecretion due to increased NF-kappa B p65, C/EBP beta, and RNA polymerase II binding to the CXCL8 promoter. J Immunol 2009; 183: 4682-4692.

20 Ogawa H, Inoue S, Ogushi F, et al. Toluene diisocyanate (TDI) nduces production of inflammatory cytokines and chemokines by bronchial epithelial cells via the epidermal growth factor receptor and p38 mitogen-activated protein kinase pathways. Exp Lung Res 2006; 32: 245-262.

21 Lee YM, Kim HA, Park HS, et al. Exposure to toluene diisocyanate (TDI) induces IL-8 production from bronchial epithelial cells: effect of pro-inflammatory cytokines. J Korean Med Sci 2003; 18: 809-812.

22 Lin CL, Zhang ZX, Xu YJ, et al. Focal adhesion kinase antisense oligodeoxynucleotides inhibit human pulmonary artery smooth muscle cells proliferation and promote human pulmonary artery smooth muscle cells apoptosis. Chin Med J (Engl) 2005; 118: 20-26.

$23 \mathrm{Li} \mathrm{K}, \mathrm{Lu} \mathrm{Y}$, Liang J, et al. RhoE enhances multidrug resistance of gastric cancer cells by suppressing Bax. Biochem Biophys Res Commun 2009; 379: 212-216.

24 Ball SG, Shuttleworth CA, Kielty CM. Platelet-derived growth factor receptor-alpha is a key determinant of smooth muscle alpha-actin filaments in bone marrow-derived mesenchymal stem cells. Int J Biochem Cell Biol 2007; 39: 379-391.

25 Foster R, Hu KQ, Lu Y, et al. Identification of a novel human Rho protein with unusual properties: GTPase deficiency and in vivo farnesylation. Mol Cell Biol 1996; 16: 2689-2699.

26 Madigan JP, Bodemann BO, Brady DC, et al. Regulation of Rnd3 localization and function by protein kinase $C$ alpha-mediated phosphorylation. Biochem J 2009; 424: 153-161. 